\title{
ON LOCALIZATION AND DOMAINS OF UNIQUENESS
}

\author{
ROE GOODMAN( $\left.{ }^{1}\right)$
}

1. Introduction. In this paper we consider the support properties of distributions $u(t), t \in R^{1}$, on an open subset $M$ of $R^{N}$ which satisfy an abstract hyperbolic equation $d u / d t=i A u$. Here $u(t)$ is assumed to be "normalizable," i.e., to belong to a Hilbert space $H$ of (vector-valued) distributions, and $A$ is a selfadjoint operator on $H$. Our general result is that if $A$ has no homogeneous Lebesgue spectrum (see $\$ 3$ for definition), then any restriction on the support of $u(t)$ for $t<0$ holds for all $t$ (Theorem 3.1), so that if the support of $u(t)$ decreases to the empty set as $t \rightarrow-\infty$, then $u=0$ (Theorem 3.2). These results generalize and sharpen theorems in [1] (some of which were also proved by a different method using energy inequalities in [4]), where a more restricted class of equations was considered and stronger assumptions on the spectrum of $A$ were made.

In $\$ 2$ we make precise the classes of equations and spaces of distributions considered. $\$ 3$ contains the main theorems on localization and domains of uniqueness, with applications in $\$ \$ 4$ and 5 . In $\S 6$ we discuss the converse of Theorems 3.1 and 3.2 , and give a counterexample.

2. Equations of evolution. Let $M$ be an open subset of $R^{N}, V$ a finite-dimensional complex vector space, and $D$ the space of $C^{\infty}$ functions from $M$ to $V$ with compact support. Give $D$ the usual locally convex inductive limit topology [5]. Suppose $H$ is a Hilbert space embedded in $D^{\prime}$, i.e., a bilinear form $\langle\cdot, \cdot\rangle$ on $H \times D$ is assumed given, such that

(A) $\phi \rightarrow\langle f, \phi\rangle$ is continuous on $D$ for each $f \in H$.

(B) $f \rightarrow\langle f, \phi\rangle$ is continuous on $H$ for each $\phi \in D$.

(C) $\langle f, \phi\rangle=0$ for all $\phi \in D$ implies $f=0$.

LeMMA 2.1. For every compact set $K \subseteq M$ there exists an integer $k$ and a constant C such that

$$
|\langle f, \phi\rangle| \leqq C\|f\|_{H}\|\phi\|_{k}
$$

for all $f \in H$ and $\phi \in D_{K}$.

Remark ON NOtation. $D_{K}$ consists of the functions in $D$ supported on $K$, $\|\cdot\|_{H}$ is the Hilbert norm on $H$, and $\|\phi\|_{k}=\sup _{|\alpha| \leqq k}\left\|D^{\alpha} \phi\right\|_{\infty}$, where $\alpha$ is a multiindex, $D^{\alpha}=D_{1}^{\alpha} \cdots D_{N}^{\alpha_{N}}, D_{j}=\partial / i \partial x_{j}$, and $\|\cdot\|_{\infty}$ is the supremum norm.

Presented to the Society, April 5, 1966; received by the editors February 23, 1966.

( $\left.{ }^{1}\right)$ Research supported in part by AFOSR and NSF contracts. 
Proof of lemma. $D_{K}$ and $H$ are Fréchet spaces, and conditions (A) and (B) state that the bilinear form $\langle\cdot, \cdot\rangle$ restricted to $D_{K} \times H$ is separately continuous. Hence by the Banach-Steinhaus theorem it is jointly continuous, from which the lemma follows.

REMARK. In particular, $H$ must be separable. Indeed, by conditions (B) and (C), the bilinear form defines a map $\pi: D \rightarrow H^{\prime}$ with $\pi(D)$ total. Hence by convexity, $\pi(D)$ is norm-dense in $H^{\prime}$. By Lemma 2.1 , if $\phi \in D_{K}$ then $\|\pi(\phi)\|_{H^{\prime}} \leqq C\|\phi\|_{k}$, from which the separability of $H^{\prime}$ and hence $H$ follows.

Suppose that $A$ is a self-adjoint operator on $H$, with domain $D[A]$, and let $W(t)$ be the one-parameter unitary group generated by $A$. Consider the abstract Cauchy problem

$$
d u / d t=i A u, \quad u(0)=f \in H .
$$

Now $u(t)=W(t) f$ is defined for any $f \in H$, and by Stone's theorem is strongly differentiable and satisfies (2.1) in case $f \in D[A]$. We shall persist in calling $u(t)$ a solution of (2.1) however, for $f$ arbitrary in $H$.

Let $u$ be a solution of (2.1), in the above sense. For each $t, u(t)$ defines via the pairing $\langle\cdot, \cdot\rangle$ a $V^{\prime}$-valued distribution on $M$. We now show that these distributions may be integrated with respect to $t$, so that $u$ defines an element of $D_{1}^{\prime}$, where $D_{1}=C^{\infty}$ functions from $M \times R^{1}$ to $V$ with compact support. Denote the points of $M_{1}=M \times R^{1}$ by $(x, t)$, and for $\phi \in D_{1}, t \in R^{1}$, let $\phi(t)=\phi(\cdot, t) \in D$.

LEMMA 2.2. For any $f \in H$ and $\phi \in D_{1}$, the function $t \rightarrow\langle W(t) f, \phi(t)\rangle$ is continuous with compact support, and the bilinear form

$$
\langle f, \phi\rangle_{1}=\int\langle W(t) f, \phi(t)\rangle d t
$$

satisfies conditions (A), (B), and (C).

Proof. For $\phi \in D_{1}$, the set $\left\{\phi(t) \mid t \in R^{1}\right\}$ is obviously bounded in $D$ (i.e., is contained in $D_{K}$ for some compact $K$ and has bounded $\|\cdot\|_{k}$ norms for all $k$ ). Also $W$ unitary implies $\left\{W(t) f \mid t \in R^{1}\right\}$ is bounded in $H$. It follows from Lemma 2.1 that $t \rightarrow\langle W(t) f, \phi(t)\rangle$ is continuous, and obviously has compact support. Furthermore, if $K_{1} \subseteq R^{1} \times M$ is compact, then there exists an integer $k_{1}$ and a constant $C_{1}$ such that

$$
\langle f, \phi\rangle_{1} \leqq C_{1}\|f\|_{H}\|\phi\|_{k_{1}}
$$

for all $f \in H$ and $\phi \in D_{1}$, supp $(\phi) \subseteq K_{1}$. This shows that the form $\langle\cdot, \cdot\rangle_{1}$ satisfies conditions (A) and (B). To verify condition (C), take $\phi \in D_{1}$ of the form $\phi(x, t)$ $=\Psi(x) \theta(t)$, where $\Psi \in D$ and $\theta \in C_{0}^{\infty}\left(R^{1}\right)$.

REMARK. By virtue of Lemma 2.2, if $u(t)=W(t) f, f \in H$, then we may consider $u$ as a distribution on $M \times R^{1}$. Thus we may speak of the support of $u$, supp $(u)$ $\subseteq M \times R^{1}$, as well as of the support of $u(t)$, supp $(u(t)) \subseteq M$, for a single value of $t$. By Lemma 2.2 and a simple approximation argument, if $\mathcal{O} \subseteq M$ is open, then $u(t)=\mathcal{O}$ on 0 for $a<t<b$ is equivalent to $u=0$ on $\mathcal{O} \times(a, b)$. 
3. Localization and domains of uniqueness. If $W(t)=\exp$ it $A$ is a one-parameter unitary group on $H$, and $f \in H$, let $\mu_{f}$ be the positive Borel measure on $R^{1}$ such that $(W(t) f, f)=\int \exp (i t \lambda) d \mu_{f}(\lambda)$. We say that $A$ has no homogeneous Lebesgue spectrum if none of the measures $\mu_{f}, f \in H$, are equivalent (in the sense of mutual absolute continuity) to Lebesgue measure on $R^{1}$. For example, if the spectrum of $A$ is a proper subset of $R^{1}$, then $A$ has no homogeneous Lebesgue spectrum.

REMARK. This condition is clearly equivalent to the condition that the representation $W$ of $R^{1}$ does not contain the regular representation.

Theorem 3.1. Let $K \subseteq M$, and let $u(t)=W(t) f, f \in H$. Suppose $A$ has no homogeneous Lebesgue spectrum. Then supp $(u(t)) \subseteq K$ for $t \leqq 0$ implies supp $(u(t)) \subseteq K$ for all $t$.

REMARK. Theorem 3.1 is also true if " $t \leqq 0$ " is replaced by " $t \leqq t_{0}$ " or " $t \geqq t_{0}$."

Proof. Let $E=\{f \in H \mid \operatorname{supp}(W(t) f) \subseteq K$ for $t \leqq 0\}$. Then $E$ is a closed subspace of $H$. Indeed, $E$ is the intersection of the null spaces of the continuous linear functionals $f \rightarrow\langle f, \Psi\rangle_{1}$, where $\Psi \in D_{1}$ and is supported on the complement of $\bar{K} \times(-\infty, 0]$ in $M_{1}$. Now $E$ is obviously invariant under $W(t)$ for $t \leqq 0$. By Theorem 1 of [2], if $A$ has no homogeneous Lebesgue spectrum then $E$ must be invariant under $W(t)$ for all $t$. Thus if $f \in E$ and $t_{0} \in R^{1}$, then $W\left(t_{0}\right) f \in E$, i.e., $\operatorname{supp}\left(W\left(t_{0}+t\right) f\right) \subseteq K$ for $t \leqq 0$. Hence $\operatorname{supp}\left(W\left(t_{0}\right) f\right) \subseteq K$. Q.E.D.

If $C \subseteq M \times R$ is open, we shall say that $C$ is a domain of uniqueness for solutions $u$ of equation (2.1) in case supp $(u) \cap C=$ void implies $u=0$. If $C$ is a domain of uniqueness, then by linearity two solutions of (2.1) which agree on $C$ are equal everywhere. Obviously any domain of the form $M \times I, I$ a nonempty open interval, is a domain of uniqueness. We establish next, using Theorem 3.1, that certain domains which are only asymptotically of this form for large negative $t$ are also domains of uniqueness, under the continuing hypothesis that $A$ have no homogeneous Lebesgue spectrum.

Notation. For $C \subseteq M \times R^{1}, t \in R^{1}$, let $C+t=\{(x, s+t) \mid(x, s) \in C\}$.

TheOREM 3.2. Let $C \subseteq M \times R^{1}$ be open, such that $C+t \subseteq C$ for $t \leqq 0$ and $\bigcup_{t>0} C+t=M \times R^{1}$. If $A$ has no homogeneous Lebesgue spectrum, then $C$ is $a$ domain of uniqueness for solutions of (2.1).

REMARK. In case $M=R^{N}$, then any open cone in $R^{N+1}=M \times R^{1}$ containing the negative $t$-axis satisfies the conditions on $C$.

Proof. Let $u(t)=W(t) f$ and suppose $\operatorname{supp}(u) \cap C=$ void. It suffices to show that $\operatorname{supp}(f) \cap \mathcal{O}=$ void for an arbitrary open set $\mathcal{O} \subseteq M$ with compact closure. Now $\overline{\mathcal{O}} \times\{0\} \subseteq \bigcup_{t>0} C+t$ and $C+t \subseteq C+s$ if $t<s$. Thus by compactness there exists a $t_{0} \in R^{1}$ such that $\mathcal{O} \times\{0\} \subseteq C+t_{0}$. Hence for any $t \leqq 0$,

$$
\mathcal{O} \times\{t\} \subseteq C+t_{0}+t \subseteq C+t_{0}
$$

so we conclude that $\mathcal{O} \times\left(-\infty,-t_{0}\right] \subseteq C$. 
By hypothesis $n$ vanishes on $C$, hence for $t \leqq-t_{0}$, supp $(u(t)) \subseteq \mathcal{O}^{\prime}$ by the above. According to Theorem 3.1, this implies that $\operatorname{supp}(u(t)) \subseteq \mathcal{O}^{\prime}$ for all $t$. Hence $f=u(0)$ vanishes on $\mathcal{O}$. Q.E.D.

4. Some applications. Let $B \geqq 0$ be a self-adjoint operator on the Hilbert space $H=L_{2}(M)$ (Lebesgue measure on $M$ ). We shall establish sufficient conditions for the preceding results on localization and domains of uniqueness to be valid for certain spaces of solutions of the abstract "wave equation"

$$
d^{2} u / d t^{2}=-B^{2} u
$$

We assume that:

$$
B^{-1} \text { exists as a bounded operator. }
$$

For $\alpha$ a real number let $B^{\alpha}$ be the nonnegative self-adjoint operator defined as usual via the spectral theorem. Define $H_{\alpha}$ to be the completion of $D\left[B^{\alpha}\right]$ with respect to the norm $\|f\|_{\alpha}=\left\|B^{\alpha} f\right\|_{L_{2}}$. (If $a \geqq 0, D\left[B^{\alpha}\right]$ is already complete in this norm, by (4.2).) Thus for $\alpha>\beta$, one has $H_{\alpha} \subset H_{\beta}$, the embedding arising from the inclusion $D\left[B^{\alpha}\right] \subseteq D\left[B^{\beta}\right]$, while $H_{0}=H$.

REMARK. In the case $B=\left(-\Delta+m^{2}\right)^{1 / 2}, m>0, \Delta$ the Laplace operator on $L_{2}\left(R^{N}\right)$ (so that equation 4.1 is the Klein-Gordon equation), the spaces $H_{\alpha}$ are well-known spaces of tempered distributions. The cases $\alpha=0$ and 1 , or $\alpha=-\frac{1}{2}$ and $\frac{1}{2}$, are encountered in the energy norm, or the Lorentz invariant norm respectively, associated with the Klein-Gordon equation.

For $\alpha \geqq 0$ the elements of $H_{\alpha}$ are $L_{2}$ functions on $M$, and thus act as distributions in the usual way. For $\alpha<0$, in order to realize $H_{\alpha}$ as a space of distributions we assume that:

(4.3 $3_{\alpha} D \equiv C_{0}^{\infty}(M) \subseteq D\left[B^{-\alpha}\right]$ and the map $B^{-\alpha}: D \rightarrow L_{2}(M)$ is continuous (relative to the topology of $D$ and the norm topology of $L_{2}$ ) with dense range.

LEMMA 4.1. Suppose $B$ satisfies (4.2) and (4.3 $)$. For every real number $\beta \geqq \alpha$, there exists a unique bilinear form on $H_{\beta} \times D$ satisfying conditions (A), (B), (C) of $\S 2$, such that for $f \in D\left[B^{\beta}\right]$ and $\phi \in D$, one has

$$
\langle f, \phi\rangle=\int_{M} f \phi d x
$$

Proof. As remarked above, the case $\alpha \geqq 0$ is trivial, the elements of $H_{\beta}$ being locally integrable functions on $M$ already. For $\alpha$ arbitrary, any such bilinear form is uniquely determined by (4.4), since $D\left[B^{\beta}\right]$ is dense in $H_{\beta}$.

Suppose $\alpha<0$. Now by the spectral theorem the operator $B$ is unitarily equivalent to a multiplication operator $M_{b}$ on a space $L_{2}(\Omega)$, where $b$ is a real-valued measurable function on $\Omega$. Let this unitary equivalence be denoted by $f \rightarrow \hat{f}$; thus $\hat{D}$ is the image of $D$ and $\hat{D}_{\beta}$ the image of $D\left[B^{\beta}\right]$. 
By assumption (4.2), $b \geqq \lambda>0$ for some constant $\lambda$. Obviously the map $f \rightarrow \hat{f}$ extends to an isomorphism of $H_{\beta}$ with the space $\hat{H}_{\beta}$ of all measurable functions $\hat{g}$ on $\Omega$ such that $b^{\beta} \hat{g} \in L_{2}(\Omega)$. In particular, if $\hat{\phi} \in \hat{D}, \hat{g} \in \hat{H}_{\beta}$, and $\beta \geqq \alpha$, then $\hat{\phi} \hat{g}=b^{\alpha-\beta} b^{-\alpha} \hat{\phi} b^{\beta} \hat{g} \in L_{1}(\Omega)$, since $b^{\alpha-\beta} \in L_{\infty}$ and $b^{-\alpha} \hat{\phi} \in L_{2}$. Define

$$
\langle g, \phi\rangle=\int_{\Omega} \hat{g}\left(\phi^{*}\right)^{\wedge} d w
$$

(the asterisk denoting complex conjugation).

Clearly if $g \in L_{2}(M)$, then $\langle g, \phi\rangle=\left(g, \phi^{*}\right)_{L_{2}}=\int g \phi d x$. Furthermore, if a sequence $\phi_{n} \rightarrow 0$ as elements of $D$, then so does the sequence $\phi_{n}^{*}$, and by (4.2) $B^{-\alpha} \phi_{n}^{*} \rightarrow 0$ in $L_{2}(M)$. Hence for $\beta \geqq \alpha, b^{-\beta} \hat{\phi}^{*} \rightarrow 0$ in $L_{2}(\Omega)$ and consequently $\left\langle g, \phi_{n}\right\rangle \rightarrow 0$ for every $g \in H_{\beta}$. Also $|\langle g, \phi\rangle| \leqq\left\|b^{\beta-\alpha}\right\|_{\infty}\|g\|_{\beta}\left\|b^{-\alpha} \hat{\phi}_{n}^{*}\right\|_{L_{2}}$, hence $g \rightarrow\langle g, \phi\rangle$ is continuous. Finally, if $g \in H_{\beta}$ and $\langle g, \phi\rangle=0$ for all $\phi \in D$, then $b^{\alpha} \hat{g} \perp b^{-\alpha} \hat{D}$ in $L_{2}(\Omega)$. But by assumption, $b^{-\alpha} \hat{D}$ is dense in $L_{2}(\Omega)$, so $b^{\alpha} \hat{g}=0$ a.e. Since $b^{\alpha}>0$ a.e., it follows that $g=0$. This establishes the existence of a bilinear form with the requisite properties. Q.E.D.

For examples of operators satisfying conditions (4.2) and (4.3 $3_{\alpha}$, we mention first the operator $B=\left(-\Delta+m^{2}\right)^{1 / 2}, m>0$, in its usual self-adjoint formulation, on $L_{2}\left(R^{N}\right) . B \geqq m$, and is hence invertible. Condition $\left(4.3_{\alpha}\right)$ is satisfied for all $\alpha<0$. Indeed, the dense range condition follows from the essential self-adjointness of $B^{-\alpha}$ on the translation-invariant domain $D$ (cf. [6]) and the invertibility of $B^{-\alpha}$; the other conditions are easily verified. Further examples arise from perturbations of the above. Suppose that $V$ is a nonnegative operator on $D$ which is of "smaller order" than $B^{2}$, i.e., there exist constants $a, b$ with $0 \leqq a<1$ such that

$$
\|V \phi\| \leqq a\left\|B^{2} \phi\right\|+b\|\phi\|,
$$

for $\phi \in D$. As is well known from the work of Kato and others, this implies that $A=B^{2}+V$ is essentially self-adjoint on $D$ (since $B^{2}$ is ess. s.a. on $D$ and $V$ has a closure, it follows that inequality (4.5) extends to all $\phi$ in $\left.D\left[B^{2}\right]\right)$. Hence the conditions (4.2) and (4.3 $)$ are satisfied for $B_{1}=A^{-1 / 2}$, with $\alpha=-2$, in which case equation (4.1) becomes $d^{2} u / d t^{2}=-\left(B^{2}+V\right) u$.

We now return to equation (4.1), and show using the spaces $H_{\alpha}$ that it can be brought to the form (2.1), by the familiar reduction of higher-order equations to systems of first-order equations.

Let $T_{\alpha}=B^{2}: H_{\alpha+1} \rightarrow H_{\alpha}$, with $D\left[T_{\alpha}\right]=H_{\alpha+2} \subseteq H_{\alpha+1}$ (i.e., in the notation of Lemma 4.1, $D\left[T_{\alpha}\right]^{\wedge}=\left\{\hat{f}\right.$ measurable on $\left.\Omega \mid b^{\alpha+2} \hat{f} \in L_{2}(\Omega)\right\}$, and $\hat{T}_{\alpha} \hat{f}=b^{2} \hat{f}$.). Then $T_{\alpha}^{*}$ is the identity map from $H_{\alpha+1}$ (with norm $\|\cdot\|_{\alpha}$ ) to $H_{\alpha+1}$ (with norm $\|\cdot\|_{\alpha+1}$ ). Thus equation (4.1) may be written as

$$
\begin{array}{ll}
d u / d t=i T_{\alpha}^{*} v, & u(0) \in H_{\alpha+1}, \\
d v / d t=i T_{\alpha} u, & v(0) \in H_{\alpha} .
\end{array}
$$


(Just as in the case of equation (2.1), this only makes literal sense when $u(t) \in H_{\alpha+2}$ and $v(t) \in H_{\alpha+1}$. We are only interested in the integrated form of the equations, however.)

Let $K_{\alpha}=H_{\alpha+1} \oplus H_{\alpha}$. Take $A$ to be the self-adjoint operator on $K_{\alpha}$ with $D[A]=D\left[T_{\alpha}\right] \oplus D\left[T_{\alpha}^{*}\right]$ and matrix

$$
\left(\begin{array}{ll}
0 & T_{\alpha}^{*} \\
T_{\alpha} & 0
\end{array}\right)
$$

relative to the given direct sum decomposition of $K_{\alpha}$. Equation (4.6) then assumes the form (2.1).

THEOREM 4.1. The conclusions of Theorems 3.1 and 3.2 hold for weak solutions $u(t)$ of (4.1), provided $u(0) \in H_{\alpha+1}, \dot{u}(0) \in H_{\alpha}$ for some real $\alpha$, and $B$ satisfies conditions (4.2) and $\left(4.3_{\alpha}\right)$.

REMARK. To be precise, $u(t)$ is assumed to be the first component of a pair $u(t) \oplus v(t) \in K_{\alpha}$, where

$$
u(t) \oplus v(t)=e^{i t A}\left(u_{0} \oplus v_{0}\right), \quad u_{0}=u(0), \quad v_{0}=\dot{u}(0),
$$

and the elements of $H_{\alpha+1}, H_{\alpha}$ act as distributions via Lemma 4.1. The dot denotes differentiation with respect to $t$ (cf. Lemma 4.2). We may assume $\alpha \leqq 0$.

Proof of theorem. By Assumption (4.2), there exists $\varepsilon>0$ such that $B \geqq \varepsilon$. A direct calculation shows that $A$ is unitarily equivalent to the operator

$$
\left(\begin{array}{rr}
B & 0 \\
0 & -B
\end{array}\right)
$$

on $K_{\alpha}$ with domain $H_{\alpha+2} \oplus H_{\alpha+1}$, and hence the spectrum of $A$ excludes the interval $\left(-\varepsilon^{\alpha+1 / 2}, \varepsilon^{\alpha+3 / 2}\right)$. In particular, $A$ has no homogeneous Lebesgue spectrum. It only remains, therefore, to show that if $\operatorname{supp}(u(t)) \subseteq K$ for $a \leqq t \leqq b$, then the same is true for $v(t)$, where $u$ and $v$ are related by (4.7). This follows from the following lemma:

LEMMA 4.2. Let $u$ and $v$ be related by equation (4.7). Then for every $\phi \in D$,

$$
d \mid d t\langle u(t), \phi\rangle=\langle v(t), \phi\rangle
$$

Proof. We may express $u(t)$ and $v(t)$ in terms of the initial data $u_{0}$ and $v_{0}$ by the familiar formulas

$$
u(t)=\cos t B u_{0}+B^{-1} \sin t B v_{0}, \quad v(t)=-B \sin t B u_{0}+\cos t B v_{0}
$$

(see [8], for example). Let $\phi \in D$. Then using the notation of Lemma 4.1, we note that $\hat{\phi} b \hat{u}_{0} \in L_{1}$, and $(d / d t) \hat{\phi} \cos t b \hat{u}_{0}=-\hat{\phi} b \sin t b \hat{u}_{0}$, pointwise on $\Omega$, with the difference quotients bounded by $3 \hat{\phi} b \hat{u}_{0}$. Hence by the dominated convergence theorem, $(d / d t) \int \hat{\phi} \cos t b \hat{u}_{0} d w=-\int \hat{\phi} b \sin t b \hat{u}_{0} d w$. A similar argument applies for the term $B^{-1} \sin t B u_{1}$, and adding yields (4.8). 
5. Half-cylinders as domains of uniqueness. In special cases, Theorem 3.1 together with standard arguments (cf. [3, Theorem 7]) can be used to obtain results on domains of uniqueness which do not require the $t$-sections of the domain to grow as $t \rightarrow-\infty$, in contrast to the domains considered in Theorem 3.1. For simplicity we shall only consider the Klein-Gordon equation. Let $B=\left(-\Delta+m^{2}\right)^{1 / 2}$, $m>0$, acting on $L_{2}\left(R^{N}\right)$, and define the spaces $H_{\alpha}$ associated with $B$ as in $\S 4$.

THEOREM 5.1. Let $u$ be a weak solution of $\square u=m^{2} u, m \neq 0$, such that for some real $\alpha, u(0) \in H_{\alpha+1}, \dot{u}(0) \in H_{\alpha}$. Suppose $\mathcal{O} \subseteq R^{N}$ is a nonempty open set such that $u(t)$ vanishes on $\mathcal{O}$ for $t \leqq 0$. Then $u=0$.

REMARKS. $\square$ denotes the operator $\Delta-\partial^{2} / \partial t^{2}$. The sense in which $u$ is a solution of the equation is that of Theorem 4.1.

Proof. By translation invariance, we may assume $\mathcal{O}$ is a neighborhood of 0 in $R^{N}$. By the results of $\$ 3$ and $4, u(t)$ must vanish on $\mathcal{O}$ for all $t$.

Now $u(t)$ is a tempered distribution with Fourier transform

$$
\cos t b \hat{u}_{0}+b^{-1} \sin t b \hat{u}_{1}
$$

where $b(\xi)=\left(|\xi|^{2}+m^{2}\right)^{1 / 2}, \xi \in R^{N}, u_{0}=u(0), u_{1}=\dot{u}(0)$, and $b^{\alpha+1} \hat{u}_{0} \in L_{2}, B^{\alpha} \hat{u}_{1} \in L_{2}$. By the vanishing of $u(t)$ on $\mathcal{O}$, if $\phi \in C_{0}^{\infty}(\mathcal{O})$, then

$$
\int \hat{\phi}\left[\cos t b \hat{u}_{0}+b^{-1} \sin t b \hat{u}_{1}\right] d \xi=0
$$

for all $t$. Let $f_{ \pm}=\frac{1}{2}\left[\hat{u}_{0} \mp i b^{-1} \hat{u}_{1}\right]$. Then $b^{\alpha+1} f_{ \pm} \in L_{2}$, and

$$
\cos t b \hat{u}_{0}+b^{-1} \sin t b \hat{u}_{1}=e^{i t b} f_{+}+e^{-i t b} f_{-} .
$$

Hence

$$
\int e^{i t b} \hat{\phi} f_{+}=-\int e^{-i t b} \hat{\phi} f_{-}
$$

Since $b \geqq 0$, the left-hand side of (5.1) is the boundary value of a function bounded and holomorphic for $\operatorname{Im} t>0$, while the right-hand side is the boundary value of a function bounded and holomorphic for $\operatorname{Im} t<0$. The equality for real $t$ thus implies by analytic continuation that each side is constant. Since $\left|e^{i t b}\right| \leqq e^{-m s}, s=\operatorname{Im}(t)$, that constant must be zero (let Im $t \rightarrow \infty$ ).

Thus we may deal with $f_{ \pm}$separately, and it clearly suffices to show $f_{ \pm}=0$. (Up to this point we have only used the positivity of the operator $B$.) Consider $f=f_{+}$(the argument for $f_{-}$is the same). Since $b^{\alpha+1} f \in L_{2}, f$ is locally integrable. Let $S^{N-1}$ be the unit sphere $\{|\xi|=1\}$ in $R^{N}$, and $d \sigma$ the invariant measure on $S^{N-1}$. For $\phi \in C_{0}^{\infty}(\mathcal{O})$, set $g(r)=\int_{S^{N-1}} \hat{\phi}(r \sigma) f(r \sigma) d \sigma, r>0$. By Fubini's theorem and the above argument, $\int_{0}^{\infty} \exp i t b(r) g(r) r^{N-1} d r=0$ for all $t$. By the change of variable $\lambda=b(r)$ this implies $\int_{m}^{\infty} e^{i t \lambda} d \mu(\lambda)=0$, where

$$
d \mu(\lambda)=g(r(\lambda)) \lambda\left(\lambda^{2}-m^{2}\right)^{N-3 / 2} d \lambda .
$$


But $\mu$ is a finite measure, and by the uniqueness of Fourier-Stieltjes transforms, $\mu=0$, hence $g=0$ a.e.

Now $C_{0}^{\infty}(\mathcal{O})$ is invariant under differentiation, hence taking Fourier transforms, we conclude that for all multi-indices $\alpha=\left(\alpha_{1}, \ldots, \alpha_{N}\right)$ and almost all $r$,

$$
\int_{S^{N-1}} \sigma^{\alpha} \hat{\phi}(r \sigma) f(r \sigma) d \sigma=0
$$

$\left(\sigma^{\alpha}=\sigma_{1}^{\alpha}{ }^{1} \cdots \sigma_{N}^{\alpha_{N}}\right.$, where $\sigma_{1}, \ldots, \sigma_{N}$ are the rectangular coordinates of $\sigma \in S^{N-1}$ ). Since $\hat{\phi} f \in L_{1}(d \sigma)$, we conclude that for almost all $r, \hat{\phi}(r \sigma) f(r \sigma)=0$ for almost all $\sigma$. Applying Fubini's theorem, we conclude that $\hat{\phi} f=0$ a.e. on $R^{N}$. Choosing a sequence $\phi_{n} \in C_{0}^{\infty}(\mathcal{O})$ such that $\hat{\phi}_{n} \rightarrow 1$ we obtain $f=0$ a.e. as desired. Q.E.D.

REMARK. Theorem 5.1 may also be proved by appealing first to the general theorem that $u$ must vanish in the full backward light cone [9, Theorem 5.3.3], and then applying Theorem 4.1 of the present paper.

6. A counter-example. The converse to Theorem 3.2, namely the presence of homogeneous Lebesgue spectrum implying the existence of nonzero solutions of (2.1) vanishing on $C$, depends on the pairing $\langle\cdot, \cdot\rangle$, i.e., the notion of localization employed. For an example where the converse does hold (cf. [3]), where nonzero finite-energy solutions to the scalar wave equation which vanish on the backward light cone are constructed. Here we give an example where it does not hold.

Let $H=L_{2}\left(R^{N}\right)$, and let $T$ be a bounded antilocal self-adjoint translationinvariant operator on $H$ (e.g., $T=(I-\Delta)^{\lambda}$ for $\lambda<0, \lambda \neq$ integer, $N$ odd (see [7])). Here antilocal means that whenever $f$ and $T f$ both vanish on a nonempty open set, then $f=0$. Let $D=C_{0}^{\infty}\left(R^{N}\right) \oplus C_{0}^{\infty}\left(R^{N}\right)$, and define a pairing on $H \times D$ by

$$
\left\langle f, \phi_{1} \oplus \phi_{2}\right\rangle=\int \phi_{1} T f d x+\int \phi_{2} f d x .
$$

Clearly this pairing satisfies conditions (A), (B), and (C) of $\$ 2$.

Let $A=\partial / i \partial x_{1}$ on $H$, so that $W(t)=e^{i t A}$ acts by translation of $x_{1}$ :

$$
W(t) f\left(x_{1}, \ldots, x_{N}\right)=f\left(x_{1}+t, \ldots, x_{N}\right) .
$$

Thus $W$ is a multiple of the regular representation of $R^{1}$ with multiplicity $\boldsymbol{\aleph}_{0}$. However, if $f \in H, f \neq 0$, then $u(t)=W(t) f$, considered as an element of $D^{\prime}$ via the pairing (6.1), has global support for all $t$, by the antilocality of $T$.

\section{REFERENCES}

1. R. W. Goodman, One-sided invariant subspaces and domains of uniqueness for hyperbolic equations, Proc. Amer. Math. Soc. 15 (1964), 653-660.

2. - Invariant subspaces for normal operators, J. Math. Mech. 15 (1966), 123-128.

3. P. Lax and R. S. Phillips, Scattering theory, Bull. Amer. Math. Soc. 70 (1964), 130-142.

4. C. S. Morawetz, A uniqueness theorem for the relativistic wave equation, Comm. Pure Appl. Math. 16 (1963), 353-362.

5. L. Schwartz, Théorie des distributions. I, Hermann, Paris, 1950. 
6. I. E. Segal, $A$ theorem on the measurability of group-invariant operators, Duke Math. J. 26 (1959), 549-552.

7. I. E. Segal and R. W. Goodman, Antilocality of certain Lorentz-invariant operators, J. Math. Mech. 14 (1965), 629-638.

8. W. A. Strauss, Scattering for hyperbolic equations. Trans. Amer. Math. Soc. 108 (1963), 13-37.

9. L. Hörmander, Linear partial differential operators, Springer, Berlin, 1963.

MassachusetTs Institute of TeChNology,

Cambridge, Massachusetts 